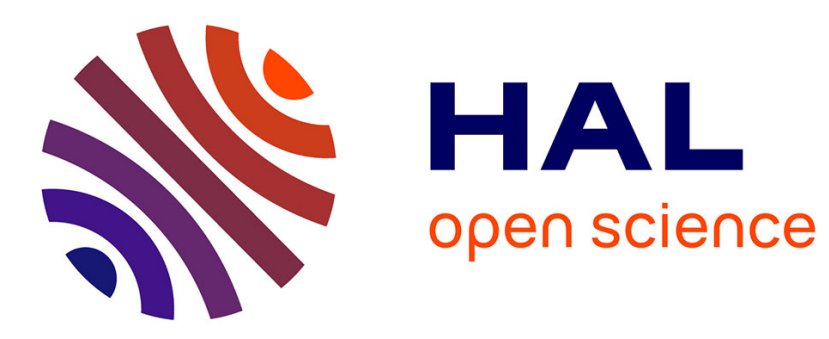

\title{
Mogwaï: a Framework to Handle Complex Queries on Large Models
}

\author{
Gwendal Daniel, Gerson Sunyé, Jordi Cabot
}

\section{To cite this version:}

Gwendal Daniel, Gerson Sunyé, Jordi Cabot. Mogwaï: a Framework to Handle Complex Queries on Large Models. RCIS 2016 - 10th International Conference on Research Challenges in Information Science, Jun 2016, Grenoble, France. pp.1-12, 10.1109/RCIS.2016.7549343 . hal-01344019

\section{HAL Id: hal-01344019 https://hal.science/hal-01344019}

Submitted on 11 Jul 2016

HAL is a multi-disciplinary open access archive for the deposit and dissemination of scientific research documents, whether they are published or not. The documents may come from teaching and research institutions in France or abroad, or from public or private research centers.
L'archive ouverte pluridisciplinaire HAL, est destinée au dépôt et à la diffusion de documents scientifiques de niveau recherche, publiés ou non, émanant des établissements d'enseignement et de recherche français ou étrangers, des laboratoires publics ou privés. 


\section{Mogwaï: a Framework to Handle Complex Queries on Large Models}

\author{
Gwendal Daniel \\ AtlanMod Team \\ Inria, Mines Nantes \& Lina \\ Gwendal.Daniel@inria.fr
}

\author{
Gerson Sunyé \\ AtlanMod Team \\ Inria, Mines Nantes \& Lina \\ Gerson.Sunye@inria.fr
}

\author{
Jordi Cabot \\ ICREA \\ UOC \\ Jordi.Cabot@icrea.cat
}

\begin{abstract}
While Model Driven Engineering is gaining more industrial interest, scalability issues when managing large models have become a major problem in current modeling frameworks. Scalable model persistence has been achieved by using NoSQL backends for model storage, but existing modeling framework APIs have not evolved accordingly, limiting NoSQL query performance benefits. In this paper we present the Mogwaï, a scalable and efficient model query framework based on a direct translation of OCL queries to Gremlin, a query language supported by several NoSQL databases. Generated Gremlin expressions are computed inside the database itself, bypassing limitations of existing framework APIs and improving overall performance, as confirmed by our experimental results showing an improvement of execution time up to a factor of 20 and a reduction of the memory overhead up to a factor of 75 for large models.

Index Terms-Model Query, OCL, Gremlin, Scalability, NoSQL.
\end{abstract}

\section{INTRODUCTION}

Model queries are a key concept in Model Driven Engineering (MDE). They constitute the basis for several modeling activities, such as model validation [1], derived features computation [2], constraint specification [3], or model transformation [4].

With the progressive adoption of MDE techniques in the industry [5], [6], existing tools have to increasingly deal with large models, and the scalability of existing technical solutions to store, edit collaboratively, transform, and query models has become a major issue [7], [8]. Large models typically appear in various engineering fields, such as civil engineering [9], automotive industry [10], product lines [11], and can be generated in model-driven reverse engineering processes [12], such as software modernization.

In the last decade, the Eclipse Modeling Framework (EMF) [13] has become the de-facto standard framework for building modeling tools, offering a strong foundation to implement model storage, querying, and persisting functionalities. The popularity of EMF is attested by the large number of available EMF-based tools on the Eclipse marketplace [14], coming from both industry and academia. Therefore, most of the research works aimed at improving modeling scalability target this framework. Nevertheless, EMF was first designed to handle simple modeling activities, and its default serialization mechanism - XMI [15] - has shown clear limitations to handle very large models [16], [17]. Furthermore, XMLbased serialization has two important drawbacks: (i) it favors readability instead of compactness and (ii) XMI files have to be entirely parsed to obtain a navigational model of their contents. The first reduces performance of I/O access operations, while the second increases the memory consumption to load and query a model, and limits the use of proxies and partial loading to inter-document relationships. In addition, XMI implementations do not provide advanced features such as transactions or collaborative edition, and large monolithic model files are challenging to integrate in existing versioning systems [18].

CDO [19] was designed to address those issues by providing a client-server repository structure to handle large model in a collaborative environment. CDO supports transactions and provides a lazy-loading mechanism, which allows the manipulation of large models in a reduced amount of memory by loading only accessed objects. Recently, the increasing popularity of NoSQL databases has led to a new generation of persistence frameworks that store models in scalable and schema-less databases. Morsa [16], [20] is one of the first approaches that uses NoSQL databases to handle very large models. It relies on a client-server architecture based on MongoDB and aims to manage scalability issues using document-oriented database facilities. NeoEMF [21] is another persistence framework initially designed to take advantage of graph databases to represent models [17], [22]. It has been extended to a multi-backend solution supporting graph and key-value stores and can be configured with application-level caches to limit database accesses.

While this evolution of model persistence backends has improved the support for managing large models, they are just a partial solution to the scalability problem in current modeling frameworks. In its core, all frameworks are based on the use of low-level model handling APIs. These APIs are then used by most other MDE tools in the framework ecosystem to query and update models. Since these APIs are focused on manipulating individual model elements and do not offer support for generic queries, all kinds of queries required by model-based tools must be translated into a sequence of API calls for individual accesses. This is clearly inefficient when combined with persistence frameworks because (i) the API granularity is too fine to benefit from the advanced 
query capabilities of the backend and (ii) an important time and memory overhead is necessary to construct navigable intermediate objects needed to interact with the API (e.g. to chain the sequence of fine-grained API calls required to obtain the final result).

To overcome this situation, we propose the Mogwaï, an efficient and scalable query framework for large models. The Mogwaï framework translates model queries written in OCL into expressions of a graph traversal language, Gremlin, which are directly used to query models stored in a NoSQL backend. We argue that this approach is more efficient and scalable than existing solutions relying on low-level APIs. To evaluate our solution, we perform a set of queries extracted from MoDisco [12] software modernization use-cases and compare the results against existing frameworks based on EMF API.

The paper is organized as follows: Section II introduces Gremlin, a language to query multiple NoSQL databases, Section III presents the architecture of our tool. Section IV and V introduces the transformation process from OCL expressions to Gremlin and the prototype we have developed. Section VI describes the benchmarks used to evaluate our solution and the results. Finally, Section VII presents related works and Section VIII summarizes the key points of the paper, draws conclusions, and presents our future work.

\section{The GREMlin QUeRY LANGUAGE}

\section{A. Motivation}

NoSQL databases are an efficient option to store large models [17], [20]. Nevertheless, their diversity in terms of structure and supported features make them hard to unify under a standard query language to be used as a generic solution for our approach.

Blueprints [23] is an interface designed to unify NoSQL database access under a common API. Initially developed for graph stores, it has been implemented by a large number of databases such as Neo4j, OrientDB, and MongoDB. Blueprints is, to our knowledge, the only interface unifying several NoSQL databases ${ }^{1}$.

Blueprints is the base of the Tinkerpop stack: a set of tools to store, serialize, manipulate, and query graph databases. Gremlin [24] is the query language designed to query Blueprints databases. It relies on a lazy data-flow framework and is able to navigate, transform, or filter a graph. It can express graph traversals finely and shows positive performance results when compared to Cypher, the pattern matching language used to query the Neo4j graph database [25].

Therefore, we choose Gremlin as our target language as it is the most mature and generic solution nowadays to query a wider variety of NoSQL databases.

\section{B. Language description}

Gremlin is a Groovy domain-specific language built on top of Pipes, a data-flow framework based on process graphs. A

\footnotetext{
${ }^{1}$ Implementation list is available at https://github.com/tinkerpop/blueprints
}

process graph is composed of vertices representing computational units and communication edges which can be combined to create a complex processing. In the Gremlin terminology, these complex processing are called traversals, and are composed of a chain of simple computational units named steps. Gremlin defines four types of steps:

- Transform steps: functions mapping inputs of a given type to outputs of another type. They constitute the core of Gremlin: they provide access to adjacent vertices, incoming and outgoing edges, and properties. In addition to built-in navigation steps, Gremlin defines a generic transformation step that applies a function to its input and returns the computed results.

- Filter steps: functions to select or reject input elements w.r.t. a given condition. They are used to check property existence, compare values, remove duplicated results, or retain particular objects in a traversal.

- Branch steps: functions to split the computation into several parallelized sub-traversals and merge their results.

- Side-effect steps: functions returning their input values and applying side-effect operations (edge or vertex creation, property update, variable definition or assignation).

In addition, the step interface provides a set of built-in methods to access meta information: number of objects in a step, output existence, or first element in a step. These methods can be called inside a traversal to control its execution or check conditions on particular elements in a step.

Gremlin allows the definition of custom steps, functions, and variables to handle query results. For example, it is possible to assign the result of a traversal to a variable and use it in another traversal, or define a custom step to handle a particular processing.

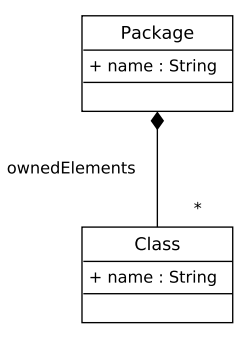

(a) Metamodel

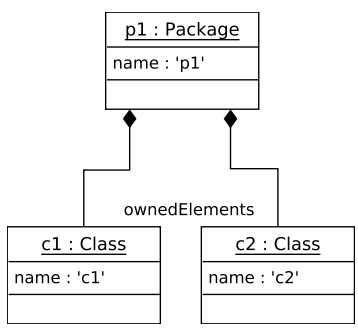

(b) Instance Model

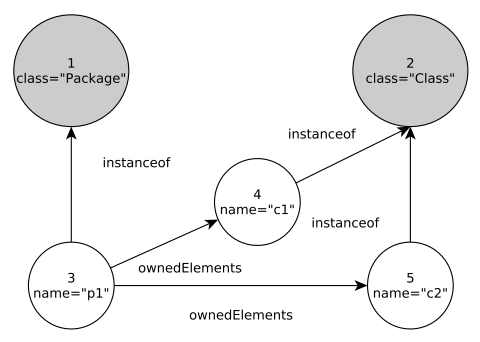

(c) Persisted Model

Fig. 1. Sample Metamodel and Model 
As an example, Figure 1(a) shows a simple metamodel representing Packages and Classes. Packages are named containers owning Classes through their ownedElements reference. An instance of this metamodel is shown in Figure 1(b) and its graph database representation is shown in Figure 1(c). Grey vertices represents Package and Class metaclasses and are linked to their instance through instanceof edges. The package $\mathrm{p} 1$ is linked to classes $\mathrm{c} 1$ and $\mathrm{c} 2$ using ownedElements edges.

In what follows, we describe some simple Gremlin examples based on this model. A Gremlin traversal begins with a Start step, that gives access to graph level informations such as indexes, vertex and edge lookups, and property based queries. For example, the traversal below performs a query on the classes index that returns the vertices indexed with the name Package, representing the Package class in the Figure 1(a). In our example, this class matches vertex 1 .

g.idx ("classes")[[name:"Package"]]; // $\rightarrow \mathrm{v}(1)$

The most common steps are transform steps, that allow navigation in a graph. The steps outE(rel) and $\mathrm{inE}(\mathrm{rel})$ navigate from input vertices to their outgoing and incoming edges, respectively, using the relationship rel as filter. inV and outV are their opposite: they compute head and tail vertices of an edge. For example, the following traversal returns all the vertices that are related to the vertex 3 by the relationship ownedElements. The Start step g.v(3) is a vertex lookup that returns the vertex with the id 3.

g.v(3).outE ("ownedElements").inV; // $\rightarrow$ [v(4),v(5)]

Filter steps are used to select or reject a subset of input elements given a condition. They are used to filter vertices given a property value, remove duplicate elements in the traversal, or get the elements of a previous step. For example, the following traversal returns all the vertices related to vertex 3 by the relationship ownedElements that have a property name with a size longer than 1 character.

g.v(3). outE("ownedElements"). inV
.has ("name"). filter $\{$ it. name. length $>1\} ; / / \rightarrow[v(4), v(5)$
]

Branch steps are particular steps used to split a traversal into sub queries, and merge their results. As an example, the following traversal collects all the id and name properties for the vertices related to vertex 3 by the relationship ownedElements. The computation is split using the copySplit step and merged in the parent traversal using exhaustMerge.

g.v(3) . outE ("ownedElements") . inV . copySplit (

-() . name,_(). id). exhaustMerge ();

Finally, side-effect steps modify a graph, compute a value, or assign variables in a traversal. They are used to fill collections with step results, update properties, or create elements. For example, it is possible to store the result of the previous traversal in a table using the Fill step.

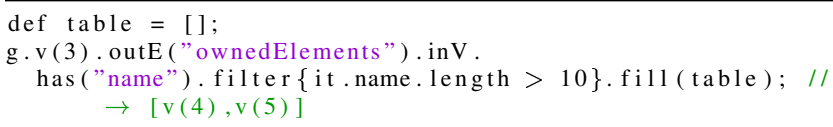

\section{The MOgWaÏ FramewORK}

The Mogwaï framework is our proposal for handling complex queries on large models. As discussed above, we will assume that those large models are stored in a NoSQL backend with Gremlin support. On the modeling side we will also assume that queries are expressed in OCL (Object Constraint Language), the OMG standard for complementing graphical languages with textual descriptions of invariants, operation contracts, derivation rules, and query expressions.

More precisely, the Mogwaï approach relies on a modelto-model transformation that generates Gremlin traversals from OCL queries which are then directly computed by any Blueprints database. The results of the query are then translated back to the modeling framework resulting in the set of modeling objects that satisfies the query expression.

Figure 2 shows the overall query process of (a) the Mogwai framework and compares it with (b) standard $\mathrm{EMF}^{2}$ API based approaches.

An initial textual OCL expression is parsed to transform it into an OCL model conforming to the OCL metamodel. This model constitute the input of a model-to-model transformation that generates the corresponding Gremlin model. The Gremlin model is then expressed as a text string conforming to the Gremlin grammar and sent to the Blueprints database for its execution.

The main difference with existing query frameworks is that the Mogwaï framework does not rely on the EMF API to perform a query. In general, API based query frameworks translate OCL queries into a sequence of low-level API calls, which are then performed one after the other on the database. While this approach has the benefit to be compatible with every EMF-based application, it does not take full advantage of the database structure and query optimizations. Furthermore, each object fetched from the database has to be reified to be navigable, even if it is not going to be part of the end result. Therefore, execution time of the EMF-based solutions strongly depends on the number of intermediate objects reified from the database (which depends on the complexity of the query but also on the size of the model, bigger models will need a larger number of reified objects to represent the intermediate steps) while for the Mogwaï framework, execution time does not depend on the number of intermediate objects, making it more scalable over large models.

Once the Gremlin traversal has been executed on the database side, the results are returned to the framework that reifies those results into the corresponding model elements. With this architecture, it is possible to plug our solution on top of various persistence frameworks and use it in multiple contexts.

To sum up, the transformation process generates a single Gremlin traversal from an OCL query and runs it over the database. This solution provides two benefits: (i) delegation of the query computation to the database, taking full advantage

\footnotetext{
${ }^{2}$ We focus the explanation on the EMF framework but results are generalizable to all other modeling frameworks we are familiar with.
} 


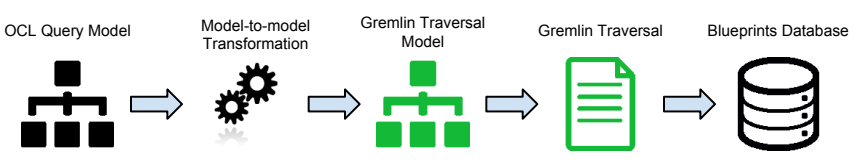

(a) The Mogwaï Query Framework

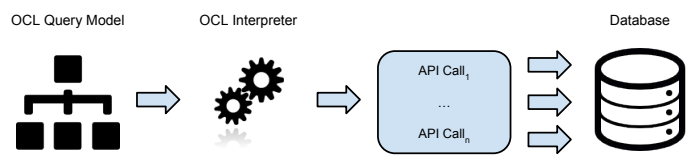

(b) EMF-based Query Frameworks

Fig. 2. Comparison of OCL execution

of the built-in caches, indexes, and query optimizers; and (ii) single execution compared to fragmented queries with the EMF API, removing intermediate object reification.

\section{OCL TO GREMLIN TRANSFORMATION}

\section{A. Mapping of OCL expressions}

To illustrate the different phases of the transformation, we introduce a running example: Listing 1 shows a simple query (on a model conforming to Figure 1(a)) that selects the Packages instances which contains at least one element through the ownedElements reference. The transformation process that generates the Gremlin traversal in Listing 2 relies on the mappings shown in Table I to translate individual OCL expressions into Gremlin steps. In this Section we detail how the different steps of the traversal are generated using this mapping. In the next Section we present how the input OCL syntax tree is processed and generated steps are linked together to produce the complete Gremlin query.

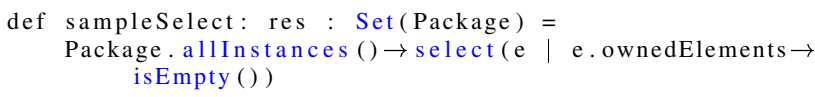

Listing 1. Sample OCL Query

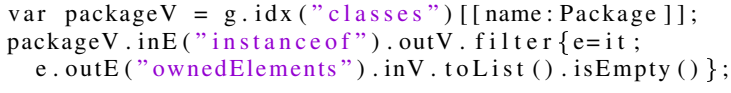

Listing 2. Generated Gremlin Textual Traversal

We have divided the supported OCL expressions into four categories based on Gremlin step types: transformations, collection operations, iterators, and general expressions. Note that other types of OCL expressions that are not explicitly listed in the table can be first expressed in terms of those that appear there [26] and therefore be also covered by our mapping.

The first group, transformation expressions, returns a computed value from their input. Expressions that navigate the elements in the model are mapped to navigation steps: Type access is translated into an index query returning the vertex representing the type, assuming the type exists. In the example, the Package type is mapped to the index call g.idx (" classes") [ [name:Package]], and the result vertex is stored in a dedicated variable to reduce database accesses. The Allinstances collection is mapped to a traversal returning adjacent vertices on the Type vertex having an instanceof outgoing edge (inE("instanceof").outV). Reference and attribute collect operations are respectively mapped to an adjacent vertex collection on the reference name and a property step accessing the attribute. Type conformance is checked by comparing the adjacent instanceof vertex with the type one using a generic transform step. Finally, attribute and reference collect from type casting are mapped as regular collect operation, because each vertex in the database contains its inherited attributes and edges.

The second group, operations on collections, needs a particular mapping because Gremlin step content is unmodifiable and cannot support collection modifications natively. Union, intersection and set subtraction expressions are mapped to the fill step, which puts the result of the traversal into a variable. We have extended Gremlin by adding union, intersection, and subtract methods that compute the result of those operations from the variables storing the traversed elements. Including operation is translated to a gather step, that collects all the objects and process the gathered list with a closure that adds the element to includes. The list is then transformed back to a step input by using the scatter step. Excluding operations can be achieved by using the except step, that removes from the traversal all the elements in its argument. A transformation of the step content into a Groovy collection is done to handle includes and excludes operations, which are then mapped to a containment checking. Finally, functions returning the size and the first element of a collection are mapped to count() and $\operatorname{first}()$ step methods. Note that there is no specific method to check if a collection is empty in Gremlin but this can be achieved by calling a Groovy collection transformation.

Iterator expressions are OCL operations that check a condition over a collection, and return either the filtered collection or a boolean value. Select is mapped to a filter step with the translation of the condition as its body. In the example the body of the select operation contains an implicit collect on the reference ownedElements and a collection operation isEmpty(), that are respectively mapped to outE ("ownedElements") . inV and toList (). isEmpty(). Reject is mapped the same way with a negation of its condition. Exists and forAll mapping follow the same schema: a filter step with the condition or its negation is generated and the number of results is analyzed. Finally, general operations (comparisons, boolean operations, variable declaration, and literals) are simply mapped to their Groovy equivalent.

The mapping presented in this Section produces all the Gremlin steps of the result traversal. In the next section, we detail the processing of the input OCL expression and how these steps are linked to produce the complete Gremlin query shown in Listing 2.

\section{B. Transformation Process}

1) OCL Metamodel: The input of the transformation is an OCL model representing the abstract syntax tree of the 
TABLE I

OCL TO GREMLIN MAPPING

\begin{tabular}{|c|c|}
\hline OCL expression & Gremlin step \\
\hline Type & g.idx('classes')[[name:'Type'] $]^{a}$ \\
\hline allinstances () & inE('instanceof').outV \\
\hline collect(attribute) & attribute \\
\hline attribute (implicit collection) & attribute \\
\hline collect(reference) & outE('reference').inV \\
\hline reference (implicit collection) & o.outE('reference').inV \\
\hline oclIsTypeOf(C) & o.outE('instanceof').inV.transform $\{$ it.next ()$==C\}$ \\
\hline oclAsType(C).attribute & attribute \\
\hline oclAsType(C).reference & outE('reference').inV \\
\hline $\mathrm{col}_{1} \rightarrow$ union $\left(\mathrm{col}_{2}\right)$ & $\operatorname{col}_{1}$. fill $\left(\operatorname{var}_{1}\right) ; \operatorname{col}_{2}$. fill $\left(\operatorname{var}_{2}\right) ;$ union $\left(\operatorname{var}_{1}, \operatorname{var}_{2}\right) ;$ \\
\hline $\mathrm{col}_{1} \rightarrow$ intersection $\left(\mathrm{col}_{2}\right)$ & $\operatorname{col}_{1}$.fill $\left(\operatorname{var}_{1}\right) ; \operatorname{col}_{2}$.fill(var2); intersection $\left(\operatorname{var}_{1}, \operatorname{var}_{2}\right)$ \\
\hline $\mathrm{col}_{1}-\mathrm{col}_{2}$ (Set subtraction) & $\operatorname{col}_{1}$. fill $\left(\operatorname{var}_{1}\right) ; \operatorname{col}_{2} . \operatorname{fill}\left(\operatorname{var}_{2}\right) ; \operatorname{subtract}\left(\operatorname{var}_{1}, \operatorname{var}_{2}\right)$ \\
\hline including(object) & gather $\{$ it $<<$ object; $\}$.scatter; \\
\hline excluding(object) & except $([$ object $])$ \\
\hline includes(object) & toList().contains(object) \\
\hline excludes(object) & !(toList().contains(object)) \\
\hline $\operatorname{size}()$ & $\operatorname{count}()$ \\
\hline first() & first() \\
\hline isEmpty() & toList().isEmpty() \\
\hline select(condition) & c.filter $\{$ condition $\}$ \\
\hline reject(condition) & c.filter $\{$ !(condition) $\}$ \\
\hline exists(expression) & filter $\{$ condition $\}$.hasNext () \\
\hline forAll(expression) & !(filter $\{$ !condition $\}$.hasNext ()$)$ \\
\hline$=,>,>=,<,<=,<>$ & $==,>,>=,<,<=, !=$ \\
\hline$+,-, /, \%, *$ & $+,-, /, \%, *$ \\
\hline and,or,not & $\& \&, \|, !$ \\
\hline variable & variable \\
\hline literals & literals \\
\hline
\end{tabular}

${ }^{a}$ Results of index queries are stored in dedicated variables to optimize database accesses

OCL query to perform. Figure 3 presents a simplified excerpt of the OCL metamodel ${ }^{3}$. In the OCL, a Constraint is a named top-level container that contains a specification described in an ExpressionInOCL element. This expression is composed of an OCLExpression representing its body, a context variable (self), and may define result and parameter variables. An OCLExpression can be a type access (TypeExp), a variable access or definition (VariableExp), or an abstract call expression (CallExp). CallExp are divided into three subclasses: OperationCallExp representing OCL operations, PropertyCallExp representing property navigations (attribute and reference accesses), and IteratorExp representing iteration loops over collections. These IteratorExp elements define an iterator Variable, and contains a body OCLExpression representing the expression to apply on each element of their input. Finally, expressions can be chained by the CallExp source reference representing the element the call apply on, or by being an argument of an OperationCallExp. In the OCL metamodel we use, all the operations are encapsulated into OperationCallExp elements. The actual identifier of the operation is contained in the name attribute.

Figure 4 shows the instance of the OCL metamodel representing the abstract syntax tree for the sample query presented in Listing 1. The top level element Constraint sampleselect contains the context variable self of the

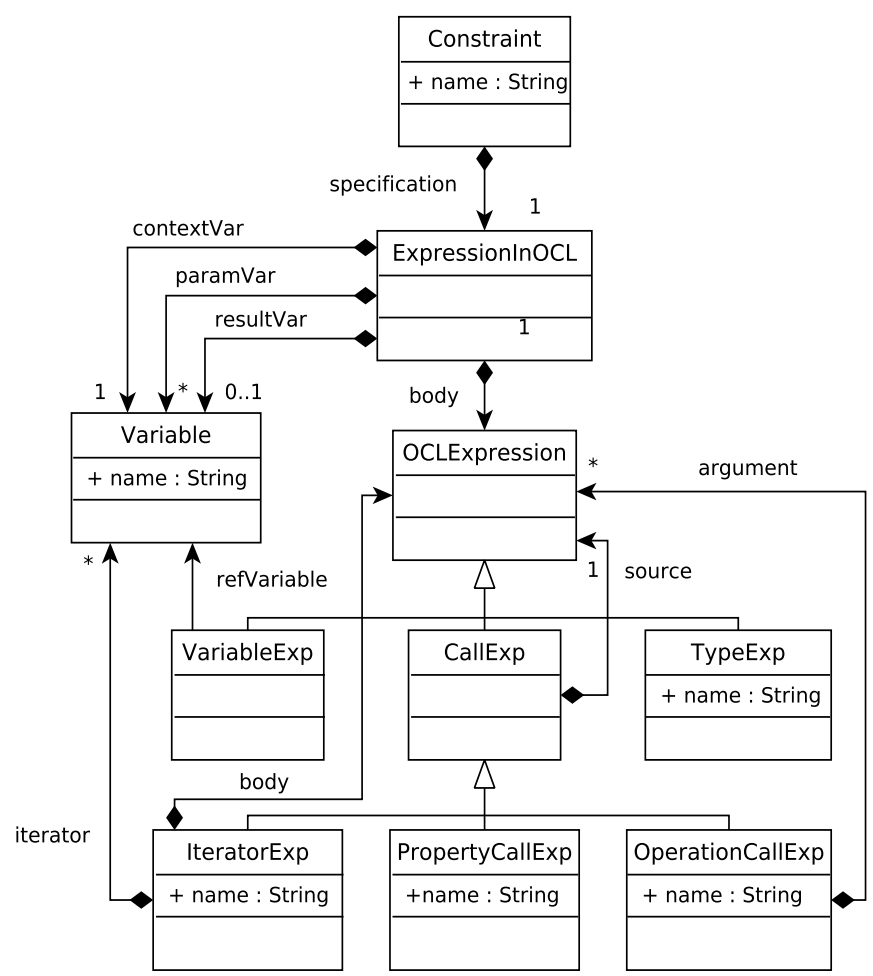

Fig. 3. Extract of OCL Metamodel

\footnotetext{
${ }^{3}$ The complete OCL metamodel we use is available at http://tinyurl.com/ hof 89 by
} 
query, its result variable (res), and an ExpressionInOCL element representing the query itself. Each expression in the OCL metamodel is linked to its source expression, in charge of computing the object/s on which the next expression will be applied. In the example the ExpressionInOCL body contains the root expression in the source tree of the query (the select in this case). This select iterator has the allinstances operation as its source, which has itself a source reference on the TypeExp Package (meaning that we iterate over the whole population of the Package class). It also defines an iterator variable $e$ and a body tree (representing the expression to evaluate over each element of the source collection) starting with the isEmpty operation that is the root of the expression. This operation is applied on the result of the ownedElements property navigation, which has a source reference to a VariableExp expression that refers to the iterator $e$.

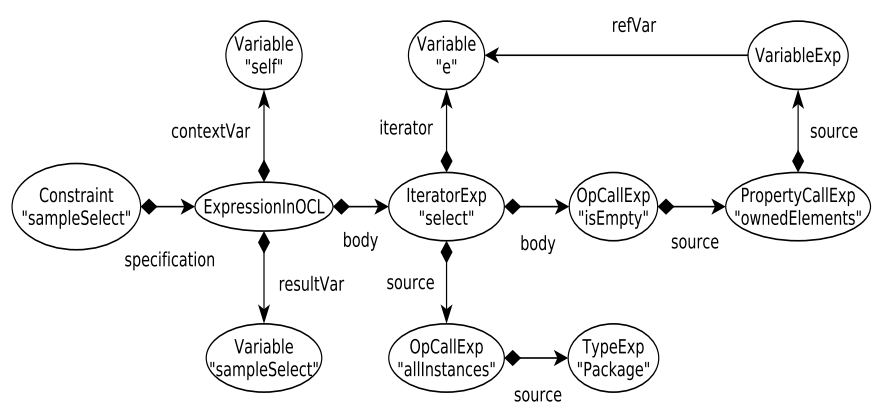

Fig. 4. OCL Query Syntax Tree

2) Gremlin Metamodel: The output of our model-to-model transformation is a Gremlin model. Since Gremlin does not have a metamodel-based representation of its grammar we propose our own Gremlin metamodel. As Gremlin is a Groovy based language, it could have been possible to reuse the Java or Groovy metamodels but they are too large for our needs and miss an easy way to define the concept of step, a core concept specific to Gremlin.

Figure 5 presents the Gremlin metamodel we use in our approach. In this metamodel, a GremlinScript is defined by a set of Instructions that can be TraversalElements, VariableDeclarations, or Expressions. Supported Expressions are unary and binary comparisons, boolean operations, and Literals. UnaryExpressions and BinaryExpressions contain respectively one and two inner Instructions. A TraversalElement is a single computation step in a Gremlin traversal. It can be either a Gremlin Step, a VariableAccess, or a MethodCall. TraversalElements are organized through a composite pattern: each Step has a next containment reference that links to the next TraversalElement in the chain. In the Gremlin terminology, such a chain of computation is called a traversal. Step elements are the core concept in the Gremlin language. We represent each step presented in Section II and the ones defined in the Gremlin documentation ${ }^{4}$ as subclasses of the Step class. Steps subclasses can contain attributes, like InEStep or OutEStep,

\footnotetext{
${ }^{4}$ http://tinyurl.com/j9hloxr
}

that contain the label of the edge to navigate. EdgesStep and VerticesStep correspond to edge and vertex lookup ( $\mathrm{g} . \mathrm{E}($ ) and $g \cdot V())$. Finally, FilterSteps are particular Steps that contain a reference to a Closure, that is defined by a set of Instructions that are applied on each filtered element.

For the sake of readability we only put the key concepts in this metamodel excerpt. In particular, we omit an important number of Steps and MethodCalls, as well as the concrete subclasses of supported unary and binary expressions. A complete definition of the metamodel is provided in the project repository ${ }^{5}$.

Figure 6 presents the instance of the Gremlin metamodel corresponding to the traversal shown in Listing 2. The toplevel GremlinScript contains two instructions. The first one is a VariableDeclaration that defines the variable packageV. The value of this variable is defined by a Gremlin traversal composed of a Start step (the initial access to the graph), an IndexCall representing the index query returning the vertex representing the metaclass Package, and a NextCall that unroll the step content and returns the vertex. The second instruction is a VariableAccess, representing the access to the variable defined in the previous instruction. This access is the begining of a second traversal composed of navigation steps (InE, OutV), and a Filter. This last step contains a Closure, representing the boolean condition of the Filter. This Closure is composed of two instructions: a VariableDeclaration that is mapped to the closure iterator, and a VariableAccess followed by a navigation, a ToList cast, and a Groovy IsEmpty check. As shown in the example, it is possible to compose Steps and regular Groovy MethodCalls in order to create a traversal.

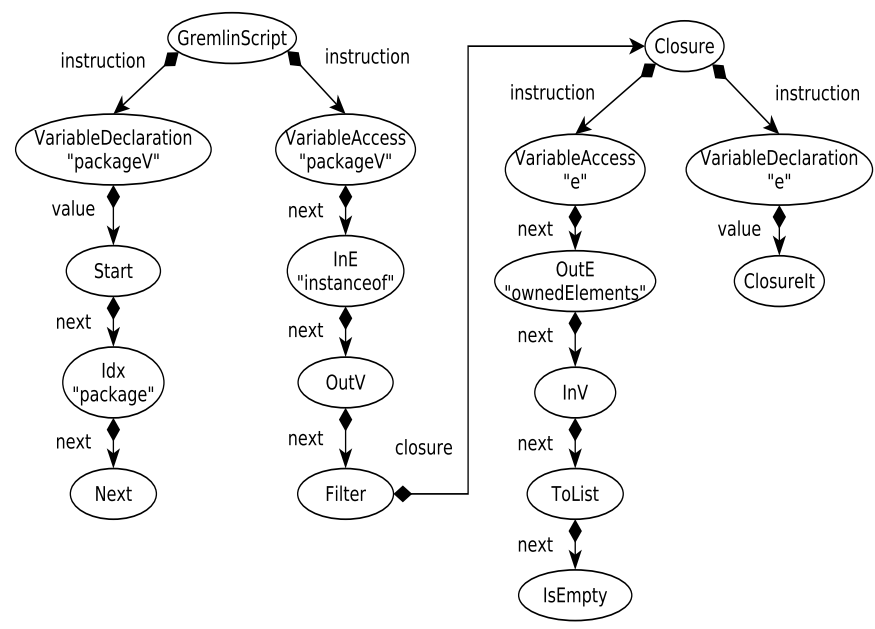

Fig. 6. Generated Gremlin Syntax Tree

3) Transformation Execution: To create a complete Gremlin traversal, the Mogwaï framework needs to process the AST model representing the syntax tree of the OCL query. In this Section we present how the input OCL query is navigated and how the different elements produced by the mappings

\footnotetext{
${ }^{5}$ http://tinyurl.com/peuyu32
} 


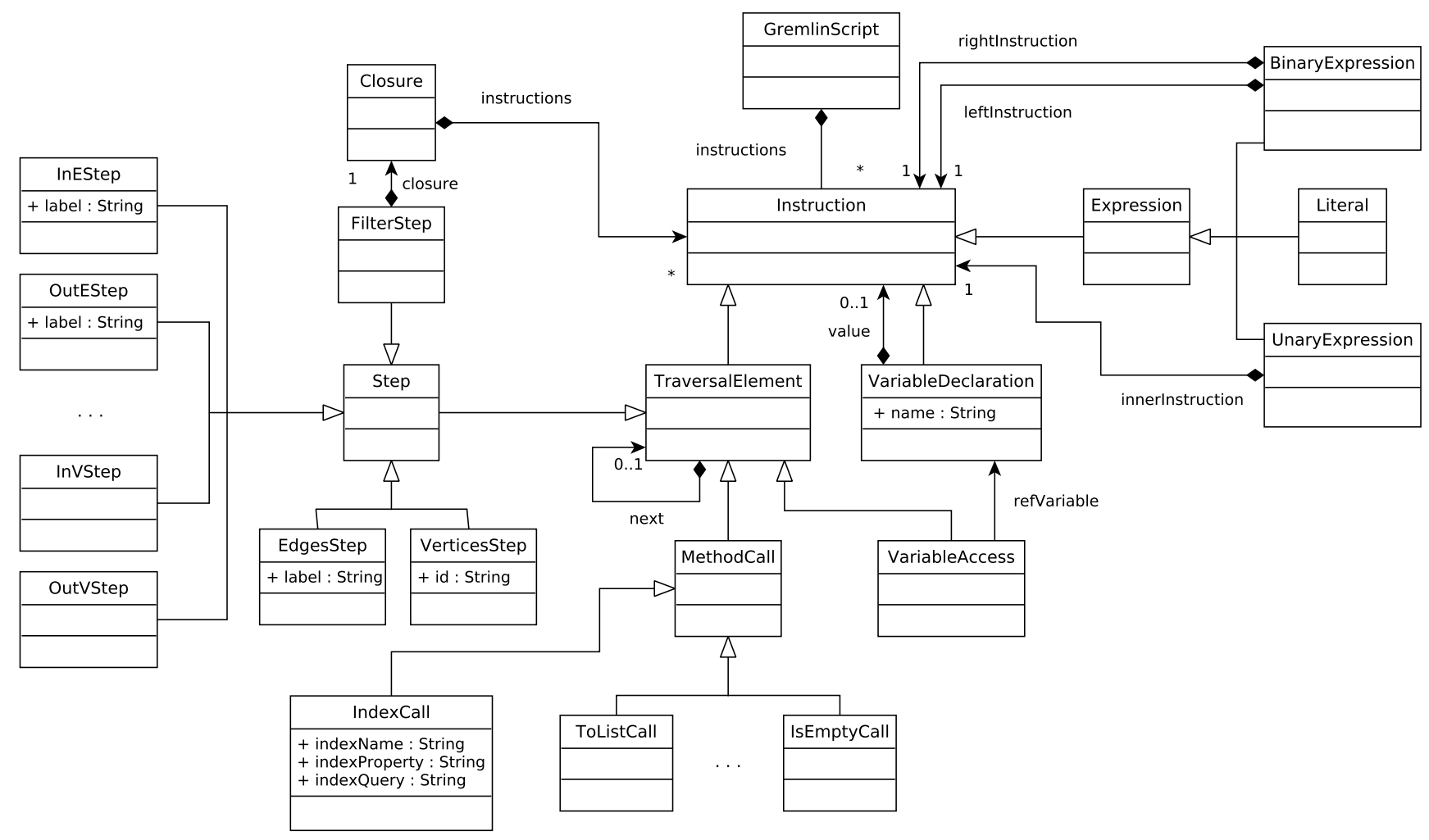

Fig. 5. Extract of Gremlin Metamodel

presented in Table I are assembled to create the final Gremlin script. For the sake of clarity, we provide an overview of the transformation in Figure 7, which presents how an input $O C L$ Query Model (1) is processed to produce the output Gremlin Traversal Model (9).

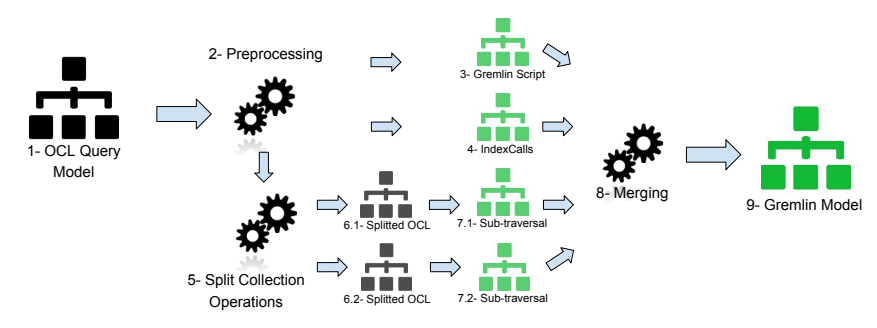

Fig. 7. Overview of the Transformation

The transformation starts by processing (2) the top-level OCL Constraint and generates the corresponding GremlinScript element (3). The input model is then inspected to find if the context variable self is accessed in the Constraint body. If it is the case, a VariableDeclaration element is created. The value of the created variable is not set at the model level, it will be binded by the framework before executing the query (see Section V). The same processing is performed to generate VariableDeclarations from parameter variables.

Once this is done, the transformation collects all the TypeExp element in the input model, and generates corresponding traversals containing an IndexCall (4) that returns the vertex from the database index representing the accessed type. The results of these calls are stored in VariableDeclaration elements. These variables are created to improve the execution performance of the generated script by caching index results and limit database access. During this step, a mapping between generated variables and TypeExp elements is computed. This mapping is then reused in the transformation to transform every TypeExp into a VariableAccess element.

In the Gremlin language, it is not possible to merge natively two traversals that do not have the same start step. Furthermore, Groovy Collection API does not provide methods to merge or subtract two collections and return the updated collection (methods such as addAll and removeAll return a boolean value, and thus can not be used as the input of the next computation step). A consequence of this limitation is that it is not possible to express in a single traversal union, intersection, and set subtraction operations. To handle these expressions, it is necessary to split the input OCL query (5) into several traversals representing each part of the operation. To handle that, the transformation collects all the root elements of each part (source and argument) of union, intersection, and set subtraction operations in the input model, and creates VariableDeclarations to store the result of the subexpressions. Each root element corresponds to an OCL expression (6.1, 6.2) that will be translated into a single traversal $(7.1,7.2)$. During this step, helper functions that compute the results of these OCL operations are also generated. 
The elements created in the different steps of the transformation are then merged (8) inside the GremlinScript to produce the output Gremlin Traversal Model (9).

To better illustrate how the transformation works, we discuss now how the Mogwaï framework transforms the OCL expression in Listing 1 to the final Gremlin expression shown in Figure 6 (abstract syntax tree) and Listing 2 (final textual expression).

As an initial step, the transformation has to preprocess the OCL model to first find the access to context and parameter variables. In our example, the input OCL expression does not contain references to those variables, and no VariableDeclaration are created. Then, the transformation collects the TypeExp Package and creates the corresponding VariableDeclaration packageV. The value of the created variable is defined by the traversal composed of a StartStep, an IndexCall, and a Next method call, representing the query performed on the class index returning the vertex corresponding to the metaclass Package.

Then, the transformation collects union, intersection, and set subtraction operations and computes their source and argument root elements. In our example, there is no such operation, and this phase simply returns the root element of the entire OCL expression.

Once this is done, we can start with the actual transformation of the OCL expressions computed in the pre-processing phase. To generate the first step of a traversal, the root expression in the source chain is retrieved and transformed according to Table I. In the example, the type access TypeExp is transformed into a VariableAccess (the one defined during the pre-processing phase). Next elements in the traversal are generated by processing the source containment tree in a postorder traversal where transformed OCL nodes are mapped and linked to the previous generated step using the next reference. In the example, this processing generates the Gremlin nodes inE('instanceof)' and outV corresponding to the allInstance expression.

Iterator operations need a particular processing: their body has to be transformed as well. In the example, the select iterator is transformed into a filter step containing a closure that represents its body. The body expression is parsed starting from the root element and generated steps are linked together. In Figure 6, body expression is mapped to variableAccess, outE('ownedElements') and inV, toList and isEmpty, corresponding respectively to the iterator access, collect(ownedElements), and isEmpty OCL expressions. The iterator Variable generates a VariableDeclaration instruction. The name of the iterator Variable is assigned to the generated VariableDeclaration, and its value contains the closure it value, that represents the current element processed. This variable shadowing is necessary to avoid it erasement in nested iterators.

Finally, if the OCL expression ends with an Union, intersection, or set subtraction operation, or if it is the last one in the argument expression, the transformation generates a Fill step that ends the traversal and puts the results in the dedicated variable defined in the initial step. Then, if the result of the operation is the source of another OCL expression, the transformation generates another traversal that starts with a MethodCall element representing the call to the helper function generated in the initial step.

To better illustrate this particular processing, we present the transformation process of the simple OCL expression shown in Listing 3.

p1. ownedElements. name $\rightarrow$ union (
p2. ownedElements. name $) \rightarrow \operatorname{size}()$ Listing 3. Sample Union OCL Query

This expression collects the names of the elements contained in the packages $\mathrm{p} 1$ and $\mathrm{p} 2$, merge them using an union operation, and returns the size of the computed collection. As stated before, the transformation starts by processing the input model in order to find source and argument root elements of the union operation. In this example, this processing returns the VariableExp $\mathrm{p} 1$ and $\mathrm{p} 2$. The transformation then generates two VariableDeclarations named union1 and union2 to store the results of the subexpressions $\mathrm{p} 1$. ownedElements . name and $\mathrm{p} 2$. ownedElements. name (line 1-2 in Listing 4). Then each subexpression is translated according to the process presented before, and the resulting traversals are affected to the generated VariableDeclaration using a Fill step (lines 3-4). In addition, the transformation generates the helper function union $(\operatorname{col} 1, \operatorname{col} 2)$ that performs the union of two collections (lines 5-7). Finally, the transformation processes the size call, that has the result of the union call as its source. A MethodCall is generated that represents a call to the helper function union, and constitute the input of the last traversal that computes the size of the collection (line 8).

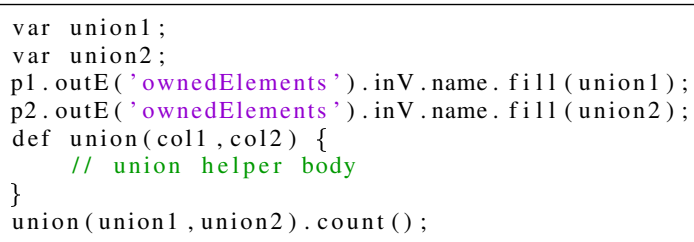

\section{Listing 4. Sample Union Traversal}

Once the traversal model has been generated, it is then parsed to produce the textual Gremlin query that is finally processed by our tool as described in the next section.

\section{TOOL SupporT}

A prototype implementation of the Mogwaï framework is provided as part of NeoEMF [17], a NoSQL persistence framework built on top of the EMF. It is implemented as an extension of the framework and supports query translation, execution, and result reification from Blueprint's persisted models. The framework presents a simple query API, that accepts a textual OCL expression or an URI to an OCL file containing the expression to transform. In addition, the query API accepts input values that represents self and parameter variables.

Initial OCL queries are parsed using Eclipse MDT OCL [27] and the output OCL models constitute the input of a set of 70 
ATL [4] transformation rules and helpers implementing the mapping presented in Table I and the associated transformation process (Section IV-B3). As an example, Listing 5 shows the transformation rule that creates a filter step from an OCL select operation. The next step is computed by the getContainer helper, which returns the parent of the element in the source tree. The instructions of the closure are contained in an ordered set, to ensure the instruction defining the iterator variable (rule var2def) is generated before the body instructions. Finally, the select body is generated, using the helper getFirstinstruction that returns the root element in a source tree.

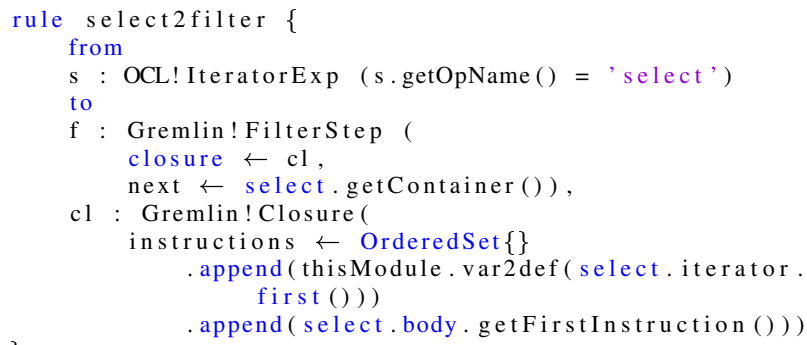

Listing 5. Select to Filter ATL Transformation Rule

Once the Gremlin model is generated by the transformation, it is expressed using its textual concrete syntax and input values corresponding to context and parameter variables are binded to the ones defined during the transformation. The resulting script is sent to an embedded Gremlin engine, which executes the traversal on the database and returns the result back to NeoEMF that reifies it to create a navigable EMF model. The reification process is done once the query has been entirely executed, and the constructed model only contains the query result objects, removing the memory overhead implied by created objects from intermediate steps of the traversal.

Finally, it is also possible to provide input elements to the Mogwaï framework to check invariants, compute a value, or navigate the model from them.

\section{EVALUATION}

In this section, we evaluate the performance of the Mogwai framework to query EMF models, in terms of memory footprint and execution time. Results are compared against performance of different querying APIs/strategies (EMF-Query, standard Eclipse OCL, IncQuery, and Mogwaii) on top of the NeoEMF/Graph backend with Neo4j.

A complementary comparison of the increased level of performance due to the uses of NoSQL solutions over SQL ones has been done in previous work [21].

Experiments are executed on a computer running Fedora 20 64 bits. Relevant hardware elements are: an Intel Core I7 processor $(2.7 \mathrm{GHz}), 16 \mathrm{~GB}$ of DDR3 SDRAM $(1600 \mathrm{MHz})$ and a SSD hard-disk. Experiments are executed on Eclipse 4.4.1 (Luna) running Java SE Runtime Environment 1.7. To run our queries, we set the virtual machine parameters -server and $-\mathrm{XX}$ :+UseConcMarkSweepGC that are recommanded in Neo4j documentation.
TABLE II

OVERVIEW OF THE EXPERIMENTAL SETS

\begin{tabular}{l|r|r|r} 
Plug-in & \# LOC & XMI Size & \# Elements \\
\hline org.eclipse.gmt.modisco.java & 22074 & $20.2 \mathrm{MB}$ & 80664 \\
org.eclipse.jdt.core & 328568 & $420.6 \mathrm{MB}$ & 1557006
\end{tabular}

\section{A. Benchmark presentation}

The experiments are run over two large models automatically generated using the MoDisco Java Discoverer. MoDisco is a reverse engineering tool able to obtain complete (lowlevel) models from Java code. The two example models are the result of applying Modisco on two Eclipse Java plug-ins: the MoDisco plug-in itself and the Eclipse Java Development Tools (JDT) core plug-in. Table II shows the details of the experimental sets in terms of number of line of code (LOC) in the plug-in, resulting XMI file size and number of model elements.

To compare the scalability of the different approaches, we perform several queries on the previous models. To simulate a realistic setting, these queries are taken from typical MoDisco software modernization use cases. Queries retrieve: ${ }^{6}$

- InvisibleMethods: collects the set of private and protected methods of a Java project.

- Grabats09: returns the set of static methods returning their containing class (singleton patterns).

- ThrownExceptions: returns the list of exceptions thrown in each package of the plug-in.

- TextElementInJavadoc: returns the textual contents of the Javadoc tags in comments of the input Model element.

- EmptyTextInJavadoc: returns the empty textual contents of the Javadoc tags in comments of the input Model element.

The first three queries start with an allinstances call, which is an important bottleneck for EMF API based query frameworks [28]. The fourth perform a partial navigation from the input Model element, and returns all the textual contents in the it javadoc comments. The last query navigates the model the same way, but only returns empty comments. Table III shows the number of intermediate objects loaded using EMF API (\#Interm.) and the size of the result set (\#Res.) to give an idea of the query complexity.

Correctness of the translation has been checked by comparing manually the results of the Mogwaï framework against results of the Eclipse OCL interpreter. In addition, we provide several test suites in the project repository ${ }^{7}$ that check the validity of the translation of single OCL expression and multiple expression composition.

All the queries are executed under two memory configurations: the first one is a large virtual machine of $8 \mathrm{~GB}$ and the second is a small-one of $250 \mathrm{MB}$. This allows us to compare the different approaches both in normal and stressed memory conditions.

\footnotetext{
${ }^{6}$ Query benchmarks can be found at http://tinyurl.com/nhpf6pq

${ }^{7}$ http://tinyurl.com/jrj66og
} 
TABLE III

Number of LoAded OBJECTS AND REsult Size For MoDisco AND JDT

\begin{tabular}{l|rr|rr} 
& \multicolumn{2}{|c|}{ MoDisco } & \multicolumn{2}{c}{ JDT } \\
& \#Interm. & \#Res. & \#Interm. & \#Res. \\
\hline InvisibleMethods & 80664 & 134 & 1557006 & 3927 \\
Grabats09 & 80664 & 0 & 1557006 & 92 \\
ThrownExceptions & 80664 & 0 & 1557006 & 1155 \\
TextElementInJavadoc & 28505 & 12359 & 136753 & 54201 \\
EmptyTextInJavadoc & 28505 & 0 & 136753 & 0
\end{tabular}

\section{B. Results}

Tables IV (MoDisco) and V (JDT) present the average results of 100 executions of the presented queries with EMFQuery, Eclipse OCL interpreter, IncQuery (detailed in Section VII), and the Mogwaï framework on a NeoEMF/Graph database. The correctness of query results has been validated by comparing the results of the different frameworks with the ones of the queries executed with the OCL interpreter.

Left columns of the tables present the time to perform the queries while right columns focus on the memory consumption implied by the query computation. All the tables present the results for large and small virtual machine configurations. Note that we were not able to express TextElementInJavadoc and EmptyTextInJavadoc queries as EMF-Query code.

\section{Discussion}

The Mogwaï framework outperforms the other query frameworks executed over NeoEMF/Graph both in terms of memory consumption and execution time. Results of all Instances based queries (1-3) show that the difference in terms of execution time is up to 20 times better than the Eclipse OCL interpreter and the EMF-Query framework, plus up to 75 times better in terms of memory consumption. This improvement is explained by (i) the absence of created intermediate objects that consume time and memory and (ii) the use of indexes and query optimizations on the database side, avoiding a complete traversal of the model elements.

Instead, if the query traverses a small subset of the model and does not use database indexes (queries 4 and 5) the benefits of using the Mogwaï framework are reduced. The result of the fourth query (where an important part of the intermediate elements are needed anyway since they are part of the result set) confirms this observation. Fourth and fifth query results also show that an important memory consumption is implied by the reification of the result elements. This overhead also impacts query execution time: execution and result reification of the fourth query over the JDT model is around $1 \mathrm{~s}$ longer than for the fifth query.

Note that the comparison only considers a single execution of each query over non-loaded models. In cases where the query is executed many times over a slightly different version of the same model, an incremental approach like the one provided by IncQuery could be a very interesting complement to our approach by using the Mogwaï framework to perform initialization queries of the incremental engine and then let the incremental engine take over from there.
To summarize these results, the Mogwaï framework is an interesting solution to perform complex queries over large models. Using query translation approach, gains in terms of execution time and memory consumption are positive, but the results also show that the overhead implied by the transformation engine may not be worthwhile when dealing with relatively small models or simple queries.

The main disadvantage of the Mogwaï framework concerns its integration to an EMF environment. To benefit from the Mogwaï, other Eclipse plug-ins need to be explicitly instructed to use it. Integration with the Mogwaï framework is straighforward but must be explicitly done. Instead, other solutions based on the standard EMF API provide benefits in a transparent manner to all tools using that API.

\section{RELATED WORK}

There are several frameworks to query models, specially targeting the EMF framework (including one or more of the EMF backends mentioned in Section I). The main ones are Eclipse MDT OCL [27], EMF-Query [29] and IncQuery [30].

Eclipse MDT OCL provides an execution environment to evaluate OCL invariants and queries over models. It relies on the EMF API to navigate the model, and stores allinstances results in a cache to speed up their computation. EMF-Query is a framework that provides an abstraction layer on top of the EMF API to query a model. It includes a set of tools to ease the definition of queries and manipulate results. Compared to the Mogwaï framework, these two solutions are strongly dependent on the EMF API, providing on the one hand an easy integration in existing EMF applications, but on the other hand they are unable to benefit from all performance advantages of NoSQL databases due to this API dependency.

EMF-IncQuery [30] is an incremental pattern matcher framework to query EMF models. It bypasses API limitations using a persistence-independent index mechanism to improve model access performance. It is based on an adaptation of a RETE algorithm, and query results are cached and incrementally updated using the EMF notification mechanism to improve performance. While EMF-IncQuery shows great execution time performances [1] when repeating a query multiple times on a model, the results presented in this article show mitigated performances for single evaluation of queries. This is not the case for our framework. Caches and indexes must be build for each query, implying a non-negligible memory overhead compared to the Mogwail framework. In addition, the initialization of the index needs a complete resource traversal, based on EMF API, which can be costly for lazy-loading persistence frameworks.

Alternatively, other approaches that target the translation of OCL expressions to other languages/technologies [31] are also relevant to our work. For example, Heidenreichin et al. [32] propose a solution to automatically build a database from a UML representation of an application, and translate the OCL invariants into database constraints. A similar approach was proposed by Brambilla et al. [33] in the field of web applications. In that case, queries are translated into triggers 
TABLE IV

Query Framework Results on MoDisco model (LARge VM / SMall VM)

\begin{tabular}{l|cccc|cccc} 
& \multicolumn{4}{|c}{ Execution Time (s) } & \multicolumn{4}{c}{ Memory Consumption (MB) } \\
& EMF-Query & OCL & IncQuery & Mogwaï & EMF-Query & OCL & IncQuery & Mogwaï \\
\hline InvisibleMethods & $9 / 9$ & $10 / 10$ & $20 / 20$ & $4 / 4$ & $25 / 21$ & $23 / 23$ & $34 / 34$ & $4 / 4$ \\
Grabats09 & $9 / 9$ & $11 / 10$ & $20 / 20$ & $4 / 4$ & $24 / 24$ & $19 / 23$ & $39 / 41$ & $4 / 4$ \\
ThrownExceptions & $11 / 11$ & $10 / 10$ & $20 / 19$ & $4 / 4$ & $23 / 24$ & $19 / 19$ & $30 / 26$ & $7 / 6$ \\
TextElementInJavadoc & $\mathrm{X}$ & $6 / 6$ & $20 / 20$ & $5 / 5$ & $\mathrm{X}$ & $10 / 9$ & $55 / 54$ & $8 / 8$ \\
EmptyTextInJavadoc & $\mathrm{X}$ & $7 / 7$ & $22 / 21$ & $4 / 4$ & $\mathrm{X}$ & $10 / 9$ & $58 / 53$ & $7 / 7$
\end{tabular}

TABLE V

QUERY FrameWORK RESUlTS ON JDT MODEL (LARGE VM / SMALL VM)

\begin{tabular}{l|cccc|cccc} 
& \multicolumn{4}{|c|}{ Execution Time (s) } & \multicolumn{3}{c}{ Memory Consumption (MB) } \\
& EMF-Query & OCL & IncQuery & Mogwaï & EMF-Query & OCL & IncQuery & Mogwaï \\
\hline InvisibleMethods & $133 / 169$ & $151 / 153$ & $326 / 662$ & $8 / 9$ & $392 / 116$ & $393 / 93$ & $550 / 162$ & $6 / 6$ \\
Grabats09 & $131 / 157$ & $154 / 158$ & $332 / 2418$ & $7 / 7$ & $388 / 120$ & $389 / 127$ & $616 / 228$ & $5 / 5$ \\
ThrownExceptions & $171 / 198$ & $151 / 157$ & $324 / 457$ & $6 / 6$ & $388 / 120$ & $386 / 92$ & $486 / 81$ & $7 / 7$ \\
TextElementInJavadoc & $\mathrm{X}$ & $19 / 20$ & $321 / 595$ & $10 / 10$ & $\mathrm{X}$ & $39 / 41$ & $570 / 171$ & $25 / 23$ \\
EmptyTextInJavadoc & $\mathrm{X}$ & $20 / 21$ & $322 / 592$ & $9 / 9$ & $\mathrm{X}$ & $42 / 40$ & $568 / 173$ & $7 / 6$
\end{tabular}

or views. Nevertheless, in all these scenarios the goal is to use OCL for code-generation purposes as part of a data validation component. Similar generative approaches exist also for other pairs of query and target languages [34]. Once generated, there is no link between the code and the models and therefore it cannot be used to speed up the model queries. In addition, all these approaches perform the translation at compilationtime, whereas the Mogwaï framework translates OCL queries to Gremlin at runtime.

De Carlos et al. [35], [36] present the Model Query Translator (MQT), an approach similar to the Mogwaï framework that translates EOL [37] queries into SQL. MQT uses a metamodel-agnostic database schema to store models, and it extends EOL to produce optimized SQL queries executed on the database side. Our translation approach is different because it relies on a model-to-model transformation to produce Gremlin traversals from OCL queries, allowing runtime execution of the transformation as well as preparation of the traversals at compilation time. In addition, graph-based navigation of models removes the overhead implied by complex joins, and the Gremlin language is expressive enough ${ }^{8}$ to translate the entire OCL.

Beyond the EMF world, proprietary meta-modeling tools provide specific query languages. This is the case of MetaEdit+ [38] from MetaCase, a commercial tool that supports the development of domain-specific languages, which provides a proprietary query language. This is also the case of ConceptBase [39], a deductive object manager for conceptual modeling and meta-modeling, which provides O-Telos, a query language for deductive databases.

Efficient model queries can also be linked to live models and Models@Run.Time [40], which aims to create adaptive software that keeps a model representation of the running system during the execution. In this environment, models become decisional artifacts that are queried during the execution

\footnotetext{
${ }^{8}$ Gremlin is written using the Groovy programming language, which is a dynamic imperative language for the Java platform
}

to take decisions, compute metrics, or retrieve information. In this context, time and memory consumption are critical aspects, since the decisions (i.e., the queries) have to be taken as quickly as possible in a stressed and concurrent environment. The results presented in this article show that the Mogwaï framework can be an interesting candidate to handle these queries both in term of memory consumption and time performance.

\section{CONCLUSIONS AND Future WORK}

In this paper we presented the Mogwaï, a framework that generates Gremlin traversals from OCL queries in order to maximize the benefits of using a NoSQL backend to store large models. OCL queries are translated using model-to-model transformation into Gremlin traversals that are then computed on the database side, reducing the overhead implied by the modeling API and the reification of intermediate objects. We also presented a prototype integrated in NeoEMF/Graph, a scalable model persistence framework that stores models into graph databases. Our experiments have shown that the Mogwaï framework outperforms existing solutions in terms of memory consumption (up to a factor of 75) and execution time (up to a factor of 20) to perform complex queries over large models.

Model transformations intensively rely on model queries to match candidate elements to transform and navigate source model. Integrating the Mogwaï framework in model transformation engines to compute these queries directly on the database could reduce drastically execution time and memory consumption implied by the transformation of large models. Another possible solution to enhance transformation engines would be to translate the transformation itself into database queries. This approach would allow to benefit of the Mogwaï framework improvements for model queries as well as element creation, deletion, or update.

As future work, we plan to study the definition of Gremlin's custom steps and to optimize collection operations to produce more readable traversals. Moreover, while the Gremlin language defines update operations, these modifications cannot 
be expressed using standard OCL, which is a side-effect free language. We plan to combine our OCL support with imperative constructs [41] allowing the efficient execution of complex update operations as well. We also plan to study the impact of semantically-equivalent OCL expressions [26] on generated traversals. With this information, it could be possible to improve the quality of the traversals by first applying an automatic refactoring on the OCL side.

Finally, we would like to study the integration of the Mogwaï framework into model persistence solutions that do not rely on a Gremlin compatible database. For instance, we plan to adapt existing work on EOL to SQL translation [35] to test our model-to-model transformation based approach over SQL databases.

\section{REFERENCES}

[1] G. Bergmann, A. Horváth, I. Ráth, and D. Varró, "Incremental evaluation of model queries over emf models: A tutorial on emf-incquery," in Proc. of the 7th ECMFA, Berlin, Heidelberg, 2011, pp. 389-390.

[2] I. Ráth, A. Hegedüs, and D. Varró, "Derived features for emf by integrating advanced model queries," in Proc. of the 8th ECMFA, Kgs. Lyngby, Denmark, 2012, pp. 102-117.

[3] OMG, "OCL Specification," 2015, URL: http://www.omg.org/spec/OCL. [Online]. Available: http://www.omg.org/spec/OCL

[4] F. Jouault, F. Allilaire, J. Bézivin, and I. Kurtev, "Atl: A model transformation tool," $S C P$, vol. 72, no. 1-2, pp. $31-39,2008$, special Issue on Second issue of experimental software and toolkits (EST).

[5] J. Hutchinson, M. Rouncefield, and J. Whittle, "Model-driven engineering practices in industry," in Proc of the 33rd ICSE. IEEE, 2011, pp. 633-642.

[6] P. Mohagheghi, M. A. Fernandez, J. A. Martell, M. Fritzsche, and W. Gilani, "Mde adoption in industry: challenges and success criteria," in Proc. of Workshops at MoDELS 2008. Springer, 2009, pp. 54-59.

[7] D. S. Kolovos, L. M. Rose, N. Matragkas, R. F. Paige, E. Guerra, J. S. Cuadrado, J. De Lara, I. Ráth, D. Varró, M. Tisi et al., "A research roadmap towards achieving scalability in model driven engineering," in Proc. of BigMDE. ACM, 2013, pp. 1-10.

[8] J. Warmer and A. Kleppe, "Building a flexible software factory using partial domain specific models," in Proc. of the 6th OOPSLA Workshop on Domain-Specific Modeling. University of Jyvaskyla, 2006, pp. 1522.

[9] S. Azhar, "Building information modeling (bim): Trends, benefits, risks, and challenges for the aec industry," Leadership and Management in Engineering, vol. 11, no. 3, pp. 241-252, 2011.

[10] G. Bergmann, Á. Horváth, I. Ráth, D. Varró, A. Balogh, Z. Balogh, and A. Ökrös, "Incremental evaluation of model queries over emf models," in Proc. of the 13th MoDELS Conference. Springer, 2010, pp. 76-90.

[11] R. Pohjonen and J.-P. Tolvanen, "Automated production of family members: Lessons learned," in Proc. of PLEES, 2002, pp. 49-57.

[12] H. Bruneliere, J. Cabot, G. Dupé, and F. Madiot, "Modisco: A model driven reverse engineering framework," IST, pp. 1012 - 1032, 2014.

[13] The Eclipse Foundation, "The Eclipse Modeling Framework," 2015. [Online]. Available: https://www.eclipse.org/modeling/emf

[14] Eclipse Foundation, "Eclipse Marketplace - Modeling Tools," 2015. [Online]. Available: http://marketplace.eclipse.org/

[15] OMG, "OMG MOF 2 XMI Mapping Specification version 2.4.1," Object Management Group, August 2011. [Online]. Available: http://www.omg.org/spec/XMI/2.4.1/

[16] J. E. Pagán and J. G. Molina, "Querying large models efficiently," IST, pp. 586-622, 2014.

[17] A. Benelallam, A. Gómez, G. Sunyé, M. Tisi, and D. Launay, "Neo4EMF, a Scalable Persistence Layer for EMF Models," in Proc. of the 10th ECMFA, York, United Kingdom, 2014, pp. 230-241.

[18] K. Barmpis and D. Kolovos, "Hawk: Towards a scalable model indexing architecture," in Proc. of BigMDE'13. New York, NY, USA: ACM, 2013, pp. 6:1-6:9.

[19] Eclipse Foundation, "The CDO Model Repository (CDO)," 2015. [Online]. Available: http://www.eclipse.org/cdo/
[20] J. E. Pagán, J. S. Cuadrado, and J. G. Molina, "Morsa: A scalable approach for persisting and accessing large models," in Proc. of the 14th MoDELS Conference, Wellington, New Zealand, 2011, pp. 77-92.

[21] A. Gómez, G. Sunyé, M. Tisi, and J. Cabot, "Map-based transparent persistence for very large models," in Proc. of the 18th FASE Conference. London, United Kingdom: Springer, 2015, pp. 19-34.

[22] G. Daniel, G. Sunyé, A. Benelallam, and M. Tisi, "Improving memory efficiency for processing large-scale models," in Proc. of BigMDE' 14 , York, United Kingdom, 2014, pp. 31-39.

[23] Tinkerpop, "Blueprints API," 2015. [Online]. Available: www.blueprints. tinkerpop.com

[24] — - "The Gremlin Language," 2015. [Online]. Available: www. gremlin.tinkerpop.com

[25] F. Holzschuher and R. Peinl, "Performance of graph query languages: Comparison of cypher, gremlin and native access in neo4j," in Proc. of the Joint EDBT/ICDT 2013 Workshops, New York, NY, USA, 2013, pp. 195-204.

[26] J. Cabot and E. Teniente, "Transformation techniques for ocl constraints," SCP, vol. 68, no. 3, pp. 179 - 195, 2007, special Issue on Model Transformation.

[27] The Eclipse Project, "MDT OCL." [Online]. Available: www.eclipse. org/modeling/mdt/?project=ocl

[28] R. Wei and D. S. Kolovos, "An efficient computation strategy for allinstances ()," BigMDE 2015, p. 32, 2015.

[29] The Eclipse Foundation, "EMF Query," 2015. [Online]. Available: https://projects.eclipse.org/projects/modeling.emf.query

[30] G. Bergmann, A. Horváth, I. Ráth, and D. Varró, "Efficient model transformations by combining pattern matching strategies," in Proc. of the 2nd ICMT, Zurich, Switzerland, 2009, pp. 20-34.

[31] J. Cabot and E. Teniente, "Constraint support in mda tools: A survey," in Proc. of the 2nd ECMDA-FA, 2006, vol. 4066, pp. 256-267.

[32] F. Heidenreich, C. Wende, and B. Demuth, "A framework for generating query language code from ocl invariants," Electronic Communications of the EASST, vol. 9, pp. 1-10, 2007.

[33] M. Brambilla and J. Cabot, "Constraint tuning and management for web applications," in Proc. of the 6th ICWE, New York, 2006, pp. 345-352.

[34] T. Halpin, M. Curland, K. Stirewalt, N. Viswanath, M. McGill, and S. Beck, "Mapping orm to datalog: An overview," in On the Move to Meaningful Internet Systems: OTM 2010 Workshops. Springer, 2010, pp. 504-513.

[35] X. D. Carlos, G. Sagardui, and S. Trujillo, "Mqt, an approach for runtime query translation: From EOL to SQL," in Proc. of OCL $2014 \mathrm{co}-$ located with MoDELS 2014, Valencia, Spain, 2014, pp. 13-22.

[36] X. De Carlos, G. Sagardui, A. Murguzur, S. Trujillo, and X. Mendialdua, "Model query translator: A model-level query approach for large-scale models," in 2015 3rd International Conference on MODELSWARD, Feb 2015, pp. 62-73.

[37] D. S. Kolovos, R. F. Paige, and F. A. Polack, "The epsilon object language (eol)," in Proc. of the 2nd ECMDA-FA. Springer, 2006, pp. $128-142$.

[38] J. Tolvanen and S. Kelly, "Metaedit+: defining and using integrated domain-specific modeling languages," in Proc. of the 24th OOPSLA Conference. ACM, 2009, pp. 819-820.

[39] M. Jarke, M. A. Jeusfeld, H. W. Nissen, C. Quix, and M. Staudt, Object Databases: 2nd ICOODB Conference. Berlin, Heidelberg: Springer, 2010, ch. Metamodelling with Datalog and Classes: ConceptBase at the Age of 21, pp. 95-112. [Online]. Available: http://dx.doi.org/10.1007/978-3-642-14681-7_6

[40] B. Morin, O. Barais, J. Jezequel, F. Fleurey, and A. Solberg, "Models@ run. time to support dynamic adaptation," Computer, vol. 42 , no. 10 , pp. 44-51, 2009.

[41] F. Büttner and M. Gogolla, "On ocl-based imperative languages," SCP, vol. 92, Part B, pp. 162-178, 2014 